\title{
Necessary aspects of quality in eLearning systems ${ }^{1}$
}

\author{
Hermann Maurer \\ Graz University of Technology \\ hmaurer@iicm.tu-graz.ac.at
}

\begin{abstract}
In this paper we discuss what is responsible for successful and high- quality eLearning. In contrast to some statements it is not the flashiness and extensive use of media, but the availability of a sophisticated e-Learning environment for administrators, teachers, tutors and students. Such a system must not only allow easy administration of all personal needs and materials involved, it must also provide a style and level that is tailored to the needs of the material and the individual involved. Based on this as starting point, it must also provide powerful collaborative features, must allow users to work with the material (not just read it) and must provide the integration of local and non-local digital libraries. Most important, eLearning must be seen as Knowledge Transfer (KT), hence not in isolation but as part of Knowledge Management (KM). Hence, techniques from $\mathrm{KM}$ have to be used, like active documents and similarity recognition, and knowledge maps, just to mention a few. We concentrate in this paper on the student view but want to caution that the administrative components of a good eLearning system very often have to be more complex than all other pieces.
\end{abstract}

Key words: WBT, eLearning, quality distance teaching, digital libraries, knowledge management, active documents, knowledge maps, similarity recognition

\section{GENERAL ASPECTS}

It is worthwhile starting with three points that we strongly believe in: First, eLearning must not be seen as tool to teach larger groups in a stratified way, but rather to provide individualized teaching at the right level of

${ }^{1}$ This paper was put in its final form while visiting Edith Cowan University, Perth, Western Australia February/March 2003. I would like to thank my host Professor Ron Oliver for continued support while working with his group at ECU.

The original version of this chapter was revised: The copyright line was incorrect. This has been corrected. The Erratum to this chapter is available at DOI: 10.1007/978-0-387-35700-3_33 
knowledge and cognitive skill of the individual student involved. This does not mean that it can't be cost effective and that it cannot be used for large groups, but it does mean that the material must adapt itself to the users: it must not provide a 'one size fits all'- kind of solution.

Second, although most members of the eLearning community have slowly started to agree that eLearning material must provide more than a slightly interactive electronic book, it is less widely understood that digital libraries (be it self-produced or purchased from publisher) can and should be used to provide important background information. Putting it differently, courseware consisting of stand-alone units that do not make use of existing digital libraries, be it local or on the WWW, provide a focus that is much too narrow.

Third, it must be recognized that no university or company can or should compete with Hollywood or TV Studios when preparing eLearning material. Good and pleasing content is necessary, but impressive multimedia material is not the answer (indeed may be distractive in some cases); the answer is the use of a suitable eLearning environment.

\section{A SUITABLE ELEARNING ENVIRONMENT}

In this section we want to address a few features of eLearning environments from a user perspective that are often not considered seriously enough. For a full set of functionalities required in a good eLearning environment see e.g. the eLearning Suite of Hyperwave (2003) or the WBTMaster (2003).

First, any good eLearning system should provide pre-tests to determine the knowledge of the student involved, and if possible also the most suitable cognitive style. Note that as far as cognitive style is concerned there is widespread agreement that the performance of learners may well depend on how material is presented, yet there are few attempts to exploit this systematically in the knowledge transfer process.

Second, a good eLearning system must support all paradigms for learning that we often hear about: no single paradigm is ideal for all applications. For fact learning, behaviourism (drill and practice) remains as valid as ever and should not be looked down upon. The cognitive approach is the most suitable, unless constructionism is a viable alternative. But there are other approaches like implicit learning (Holzinger et al 2001), situation learning (Maurer and Pivec 2001), etc. that also must not be ignored.

Third, users should be able to work with the material to an extent that goes beyond all learning theories: we will return to this in the next section. 
Fourth, when eLearning is used in a networked environment (and notnetworked attempts have been successful only in very isolated cases) the network must be exploited for communication and collaboration to the fullest, including discussion forums, chats, shared work spaces and the like. Note in particular that although discussion forums are already in widespread use, such forums are rarely powerful enough to handle large-scale discussions, including the re-structuring of discussions, the merging (!) of discussions or the extraction of parts of a discussion as a special resource to be used elsewhere. There are systems with powerful discussion forums such as eLS (2003) and WBT-Master (2003), but some basic rules have already been formulated much earlier (Maurer et al 1999).

Fifth, any kind of courseware or teachware should not be seen in isolation, but always in conjunction with sufficiently large digital background libraries. Such libraries can consist of material generated in the course of other activities, or can be purchased in the form of libraries available on the WWW or material on CDs and DVDs. As is pointed out by Maurer and Tochtermann (2002) using techniques from knowledge management that will be discussed in a later section, automatically generated links, even ones that can be visualized as 'knowledge maps' such as the ones in Der Brockhaus (2001) should and can be used. For some aspects of digital libraries and their use consult Marchioni and Maurer (1998), Lennon and Maurer (1995) and Maurer (2001).

\section{WORKING WITH MATERIAL}

It is generally accepted that passively observing material on a computer screen, no matter how many pictures, diagrams, animations, movies, audio material etc. are used is not enough to create a productive learning situation. Much more interaction is essential. This is, after all, the basis of many learning theories. Only by letting students solve problems, collaborate with each other etc. will they be sufficiently involved in a process conducive to serious learning.

However, what is often overlooked is that students should also be allowed to 'massage' material that is presented to them, by adding notes, adding links to the WWW or a background library, by attaching files, by highlighting, supplementing or erasing parts of what is shown to them etc. In each case, such changes will either be just for one student (producing a particular student's view) or for a group (producing a special view of the group collaborating). It is our experience that in this way material offered to students will expand, different persons or groups of persons ending up with often surprisingly different versions of the original teaching material (which 
of course is never modified as such: the modifications are only superimposed and only visible to those who have authority to see them: noone except for the original author can change the underlying substance.)

\section{WHY COMMUNICATION IS IMPORTANT}

Communication is not just important to break the isolation of students in an eLearning environment but also for a much more basic reason: whatever one person says or writes, the receiver of the information will always interpret the information in the receiver's personal context, created through upbringing, culture, language, etc. This often leads to deep misunderstandings. Our favourite example is the story of a fish who, when hearing of a flying animal does not think of a bird as we know it, but of course of a fish with wings; or when hearing of a 'four legged animal with an udder with milk' is more likely to imagine a frog with an udder with milk than a cow, simply because frogs are probably the only four legged creatures fish know.

It is often claimed that a picture says more than a thousand words and this may well be true. Although, at times, misconceptions might be resolved using pictures, this is by no means always the case: a fish that happens to see a person drinking a glass of water will be quite dumbfounded, for such action does not seem to make sense to the fish; a nomadic person in the dessert (who has never seen anything but the dessert) will not understand the picture of fog rising over a lake, nor will the traditional Indian in the Amazon jungle be able to make much sense of trees covered with lots of snow when shown a picture of a winter scene from Austria.

The only way to make sure that information is properly understood is not by reading, hearing, seeing, but by being able to check if things have been understood and by asking questions: this is why an eLearning system that ignores the importance of communication will not work.

There is one more subtle aspect about communication: we believe that communication should not be restricted to communication between persons but should be extended to cover communication between students and documents. To formulate it in an exaggerated way: we would like to see systems where a student who sees something on the screen can type in any question whatsoever and the document (!) gives the answer. Although this sounds like an absolute impossibility, the situation described can be approximated quite well if the information on the screen is viewed by many (thousands!) of persons before it changes. In this case the concept of 'active documents' (Heinrich and Maurer, 2000 and Heinrich et al, 2001), can be applied: when the first few hundred users ask questions, the answers are 
given by experts, but both questions and answers are stored in a database. Later questions that can be recognized to be semantically identical with earlier ones by the system can then be answered by the system, i.e. the documents. In large applications we have found that over $99.5 \%$ (!) of all questions can indeed be answered without human intervention. The 'Knowledge Information Centre' of Hyperwave (2003) is one such software module. Indeed, many of the features discussed so far, and including the knowledge management aspects to be discussed in the next section have been successfully handled by extending Hyperwave. For background information consult Maurer 1996 and Maurer 1998.

\section{KNOWLEDGE MANAGEMENT}

Teaching and learning clearly involve Knowledge Transfer (KT), and hence eLearning is clearly a small subset of the fairly new but increasingly important area of Knowledge Management (KM). Thus, eLearning must not be dealt with in isolation but with at least a minimum of information about $\mathrm{KM}$ and its tools. Rather than defining KM, it is easier to explain KM by quoting the famous statement: 'If our employees only knew what our employees know we would be a much better organisation.'

Thus, the original challenge of $\mathrm{KM}$ is to extract knowledge from persons (without burdening them with extra work), storing the information in a computer system, and making the knowledge available to users when they need it (even if they have not asked for it). It is exactly the two parenthesised remarks in the last sentence that distinguish KM systems from ordinary information systems or databases: in ordinary information systems, information has to be input and requested explicitly. Surprising as this may sound, the automatic extraction of knowledge without imposing extra work on the persons whose knowledge is desired, and the provision of relevant information at the right moment is indeed possible to an increasingly high percentage. For details we refer to Maurer and Tochtermann, (2002) and the references therein, e.g. Ives et al (1998), Meersmann et al (1999). However we would like to at least mention three of the many tools that are currently used in KM that directly apply to eLearning: the first two, 'knowledge maps' and 'active documents' we have already briefly mentioned above. And the active document concept contains in it the seed for something much larger: after all, to discover if two questions are semantically the same, one basically needs mechanisms to discover if two documents are similar.

It is this similarity- recognition that proves to be invaluable in $\mathrm{KM}$ and in eLearning. Let us look at a number of simple examples to try to prove our point, the first one from the commercial world, the others from eLearning. 
In a large, world-wide distributed manufacturing company a new project is to be undertaken. The engineers draw up, according to quality assurance procedures, a detailed description of the product in a fairly standardized manner. The Hyperwave system that we have installed in one such instance translates the specifications from whatever language into (rather mediocre quality) English. This English document is compared to the English descriptions of all projects, planned or in progress in all locations of the company at issue. If it detects a strong similarity, it alerts both groups involved to avoid potential duplication. This 'simple' procedure has saved the company at issue more than ten million US\$ in a single year!

Suppose you are writing a paper. As soon as you have finished your extended abstract you switch your system into supervisory mode. It now starts to check what you have written against all material available in background libraries world- wide. If it finds some 'suspicious' similarity, it will alert you: you might be frustrated since your 'novel idea' turns out to be not quite as novel as you thought (but better you find out now than later!) but it may also help you to show you results you can put to good use.

There are many applications that directly apply to eLearning. Similarity recognition (SR) may alert a student to another student who is doing similar work. SR may help students to find fellow students who are experts in topics they are currently interested in. SR may help a teacher to find out an incident of plagiarism. SR can help to short-cut discussions in forum by pointing out that the same topic has already been treated exhaustively some time back, etc.

Without going deeper it should be clear by now that tools developed for $\mathrm{KM}$ are very much applicable to eLearning, and must not be ignored by the eLearning community as has largely been the case with some exceptions like Hyperwave(2003) and WBT-Master (2003) mentioned earlier.

\section{THE IMPACT OF NEW TECHNOLOGY ON ELEARNING}

Laptops are becoming lighter and lighter, hand-helds get more sophisticated, the mobile phone has increasingly powerful computing resources. Yet this is very much the beginning of an era that will start around 2010. At that point mobile phones will have turned into veritable computerpowerhouses, let us call them PC2010: not much bigger than a credit card, with a fast processor, gigabytes of internal memory, a combination of mobile-phone, computer, camera (still and video), global positioning system, a variety of sensors and in continuous connection with huge non-volatile 
local storage and the then existing equivalence of the internet: continuous, since there will be no charge for connect time, just for data transfer. Most important, the PC 2010 will have no hard-disk: this fragile energy consuming device with rotating parts will be replaced by a version of the memory stick as we now use them in digital cameras, but with hundreds of Gigabyte capacity; it will have no screen nor keyboard as we now have it, and the much smaller energy needed because of all this will be provided by tiny fuel-cells.

Of all of the above we believe that you might be startled by only two things: the promised absence of screen and keyboard. Let us first address the issue of screen.

Presently, there are half a dozen technologies competing to replace the screen as we now have it. They include flexible screens that can, for example, be attached to your sleeves, projectors that create images wherever you want (even on uneven surfaces of any colour), and the spectrum continues to specialized glasses, just to mention three alternatives. Which of those technologies will 'win we do not know, nor does it matter: what matters is that wherever you go you will have a more or less zero-weight high quality display at your disposal, connected to the small computer proper by the modernized version of blue-tooth, and via the computer to a huge archive of information locally and all the servers on the internet. Of the screen technologies possible we particularly like a version of eyeglasses, maybe because we have already used a prototype: the electronics in the eyeglasses are in contact with the computer via blue-tooth. The computer delivers sound, stereo if required, to the side of the glasses, that transmit it directly to the ear-bones (thus, only the wearer can hear the signals); the computer transmits (moving or still) pictures (if wanted 3D) through little mirrors through the pupils of the eyes directly to the retinas; and a tiny camera in the middle of the glasses provides the computer with what the user sees, e.g. for gesture recognition.

Indeed, the PC2010 is capable of doing much more (like sophisticated image processing of the pictures obtained by the camera) but this is the subject of another forthcoming paper (Maurer 2003).

Let us now turn to the keyboard. First, alternative input techniques are starting to emerge. Speech input is one of them, and is particularly attractive if 'speech that is not heard' is used (i.e. utterances with closed mouth), e.g. using microphones near the larynx. Second, techniques that use the movement of fingers, the head, or the body using tiny sensors are becoming realistic; third, using the glasses with integrated camera described above a 'virtual keyboard' can be made visible to the user, and the finger movements on that keyboard can be analysed by software image-processing what the camera delivers. 
Lest we are misinterpreted: we are not betting our money on any of the technologies mentioned, but we are betting our money on the fact the screens, hard-disks and keyboards will be obsolete within ten years, give or take a few years.

However, such a PC2010 implies that (if a good KM system is available (Hypermedia 2003, Maurer and Tochtermann 2002) most of our knowledge will reside outside our brain, no need to store it inside (!), hence learning will change dramatically. We find it curious that the question HOW we teach in the future is still considered more important than the question WHAT we teach in the future, since this is the area with the most dramatic changes. Clearly, much factual knowledge (that professions like lawyers, medical persons, geographers, and, and, and) are currently cramming into their heads during their studies will be quite superfluous.

Let us finish with one provocative example: we are still teaching children of age six or so handwriting. Who believes that this will be a necessary skill when those children leave school in ten years or more? WE see a high probability that such a skill is going to be as outdated as the use of logarithm tables in mathematics is outdated today. Yet we are not even discussing the issue!

To be objective, it may well be that teaching handwriting should survive: not because the skill as such is needed, but because it is one way of fostering hand-eye coordination. However, in that case, should we not start to investigate whether juggling is even better for training hand-eye coordination?

To put the last thoughts in one sentence: we urge the eLearning community not only to investigate HOW to teach in the future, but to put more emphasis on thinking about WHAT we should teach, assuming the technological speculations in this paper are not too far off?

\section{REFERENCES}

Der Brockhaus Multimedial (2002) Premium; DVD, Brockhaus Verlag, Mannheim (2001).

ELS (2003) www.hyperwave.de

Heinrich, E. Maurer, H. (2000). Active Documents: Concept, Implementation and Applications. J.UCS 6, 12, 1197-1202.

Heinrich, E., Johnson, R. Luo, D., Maurer, H. (2001). Learner-Formulated Questions in Technology-Supported Learning Applications; Proceedings of ED-MEDIA 2001, AACE, Charlottesville, VA, 720-725.

Holzinger, A., Pichler, A., Almer, W., Maurer, H. (2001). TRIANGLE: A multi-Media testbed for examining incidental learning, motivation and the Tamagotchi-Effect within a Game-Show like Computer Based Learning Module; Proceedings of ED-MEDIA 2001, AACE, Charlottesville, VA, 766-771.

Hypermedia (2003) http://www.hyperwave.com 
Ives, W., Torrey, B., Gordon, C. (1998). Knowledge Management: An emerging discipline with a long history; Journal of Knowledge Management 1, 4 , 269- 274.

Lennon, J., Maurer, H. (1995). Digital Libraries as Learning and Teaching Support; J.UCS 1/11, 719-727; Proc. ICCE'95, Singapore, AACE, Charlottesville, USA, 28-33.

Marchionini, G., Maurer, H. (1995). The roles of digital libraries in teaching and learning; C.ACM 38, 4), 67-75.

Maurer, H. (1996). Hyperwave - the Next Generation Web Solution; Addison Wesley Longman.

Maurer, H. (1998). Using the WWW System Hyperwave as the Basis of a General Networked Teaching and Learning Environment; CIT, Vol. 6, 1 (special issue).

Maurer, H., Rozsenich, N., Sapper, M. (1999). How to Make Discussion Forums Work on the WWW; Proc. WebNet 99, AACE, Charlottesville, USA, 717-722.

Maurer, H., Pivec, M. (2001). Situation Learning: A New Approach to Knowledge Mediation; Proceedings of ED-MEDIA 2001, Tampere, AACE, Charlottesville, VA, 1254-1259.

Maurer, H. (2001). Beyond classical digital libraries Global Digital Library Development in the New Millenium; Proceedings NIT Conference, Beijing, Tsinghua University Press, 165-173.

Maurer, H., Tochtermann, K. (2002). On a New Powerful Model for Knowledge Management and its Applications; JUCS 8, 1, 85-96.

Maurer, H., Oliver, R (2003):: A scenario for the computer 2010 and its implications for society; forthcoming paper, consult www.iicm.edu/maurer for details

Meersman, R., Tari, Z., Stevens, S. (Eds.) (1999). Database Semantics; Kluwer Academic Publishers, USA.

WBT-Master (2003) http://coronet.iicm.edu 